\title{
Multi-spacecraft observations of polar coronal plumes
}

\author{
W. Curdt ${ }^{1}$, K. Wilhelm ${ }^{1}$, L. Feng ${ }^{1}$, and S. Kamio ${ }^{2}$ \\ 1 Max-Planck-Institut für Sonnensystemforschung (MPS), Max-Planck-Str.2, 37191 Katlenburg-Lindau, Germany \\ e-mail: curdt@mps.mpg.de \\ 2 National Astronomical Observatory of Japan (NAOJ), Mitaka, Tokyo 181-8588, Japan
}

Received 14 November 2007 / Accepted 14 January 2008

ABSTRACT

\begin{abstract}
Aims. Coronal plumes, along with their structure and rôle in the acceleration of the fast solar wind, are of considerable importance in understanding the physics of the solar atmosphere, in particular, when contrasted with the adjacent plasma, the so-called interplume regions.

Methods. Observations of coronal plumes in a coronal hole were obtained with the help of spectrographs and imagers on Hinode, SOHO, STEREO, TRACE, and with ground-based instrumentation. The measurements were performed by a large international team in April 2007.

Results. First results of this study from Hinode, SOHO, and STEREO are presented here, at the level of observational facts, leaving the task of detailed analysis to the future.
\end{abstract}

Key words. Sun: corona - Sun: solar wind - techniques: spectroscopic

\section{Introduction}

Polar coronal plumes seen during solar eclipses can now be studied with space-borne telescopes and spectrometers. The sources of these plumes are still not fully identified, and it is being debated whether they contribute substantially to the fast solar wind (DeForest et al. 1997; Wilhelm et al. 1998; Teriaca et al. 2003; Gabriel et al. 2003; Antonucci et al. 2004). An understanding of the processes of plume formation requires better knowledge of the physical conditions in plumes and the surrounding interplume environment. Specifically, the electron densities and temperatures, the effective ion temperatures and non-thermal motions, the plume cross-section relative to the size of the coronal hole $(\mathrm{CH})$, and the plasma bulk speeds, all have to be known. Some of these aspects have motivated our work here.

\section{Multi-spacecraft observations}

Early in April 2007, the southern polar coronal hole was well developed; and since the South pole was tipped towards the Earth with a pole angle of $6.2^{\circ}$, it was decided to observe this region. The X-Ray Telescope (XRT, Golub et al. 2007) and the EUV Imaging Spectroscope (EIS, Culhane et al. 2007) on Hinode (Kosugi et al. 2007) observed the $\mathrm{CH}$ on the disk, while SUMER on $S O H O$ obtained a large raster of $300^{\prime \prime} \times 600^{\prime \prime}$ above the limb. Images taken by the Extreme UltraViolet Imager (EUVI) instruments on STEREO A and B (Wülser et al. 2007) have been analysed to provide $3 \mathrm{D}$ information about the plumes.

\subsection{Observations by EIS and XRT}

Several coronal bright points (BP), which are thought to be the footpoints of plumes (Ahmad \& Withbroe 1977; Lemaire et al. 1997; Wang et al. 1997), are seen in soft X rays by XRT and in Fe XII $19.5 \mathrm{~nm}$ emission by EIS. They allow an exact co-alignment. The soft X-ray image in Fig. 1 was obtained by XRT at 22:39 UTC on April 7. The plus signs indicate locations of plume footpoints determined from EUVI (see Sect. 2.2). Two footpoints around $x=-80^{\prime \prime}$ are close to BPs. However, no prominent $\mathrm{BP}$ is found near another plume footpoint at about $x=70^{\prime \prime}$. The brightness enhancement at $x=100^{\prime \prime}$ is behind the limb. The BP on the limb at $x=-80^{\prime \prime}$ attained its peak radiance at that time, but underwent transient brightenings on typical time scales of $10 \mathrm{~min}$, as shown in the lightcurve in Fig. 2. Some of these brightness enhancements were associated with X-ray jets. This interesting aspect is not relevant for this communication but is discussed elsewhere (e.g., Cirtain et al. 2007).

Figure 3 shows a radiance map in the spectral line Fe XII $19.5 \mathrm{~nm}$ obtained with EIS. Although this image is not a snapshot - it took $5 \mathrm{~h}$ to complete the raster scan - quasistationary plume structures are clearly seen above the limb. Two of the EUVI footpoints were covered by the scan. The plume is seen as a weak enhancement in Fe XII emission, since the formation temperature of Fe XII $(\approx 1 \mathrm{MK})$ is above the plume plasma temperature. Note that the distribution of BPs is different from what is shown in Fig. 1, because of the time difference between the data. The EIS scan was run from 00:22 to 05:48 UTC many hours before the XRT image was taken. Sequences of XRT images (not shown) indicate that BPs, in addition to their short-term variability mentioned above, vary substantially on time scales of hours, while plume structures are known to be quasi-stationary and can last for one day or more.

\subsection{EUVI observations}

Stereoscopic images (obtained on April 7 at 22:07 UTC) in the $17.1 \mathrm{~nm}$ passband of the EUVI instruments on the STEREO A 


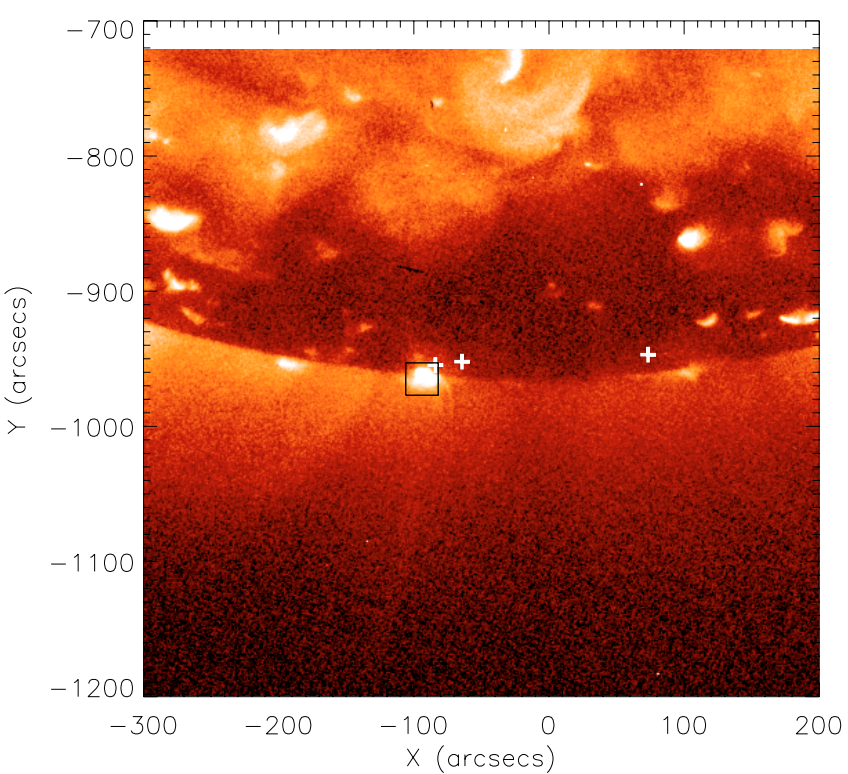

Fig. 1. Soft X-ray image obtained with the Al-poly filter of XRT. Radiances are on a logarithmic scale and plus signs in the plot mark the location of plume footpoints (cf., Fig. 4). The lightcurve of the BP marked with a box is shown in Fig. 2.

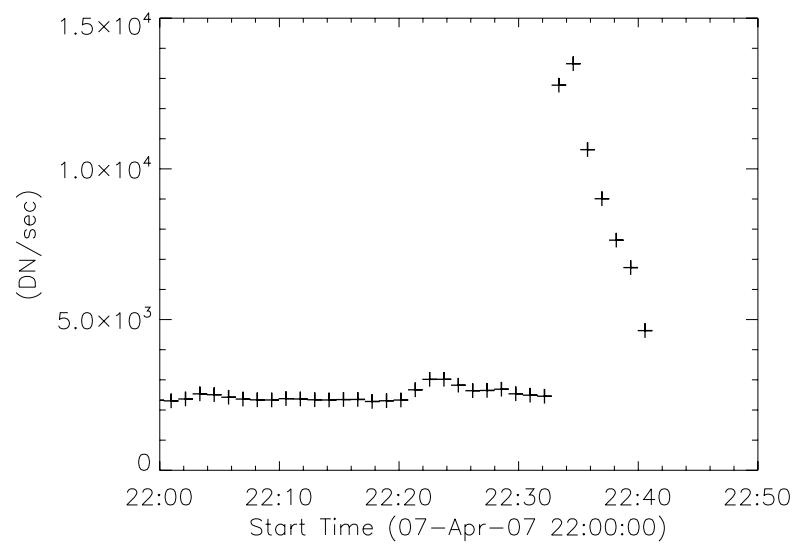

Fig. 2. Soft X-ray lightcurve of the bright point marked with a box in Fig. 1. A sudden burst started at 22:33 and lasted until the end of the observation, i.e. at least for $8 \mathrm{~min}$.

and B spacecraft were used to reconstruct the 3D geometry of five plumes as shown in Fig. 4. The formation temperatures of the Fe IX and FeX lines in this band $(\leq 1 \mathrm{MK})$ are ideal for coronal-hole observations. Linear fits outline the plume structures in epipolar view and out-of-plane distances from the footpoints up to $0.15 R_{\odot}$ are coded by the symbol size.

The reconstruction is based on displacements of identifiable structures seen in stereoscopic view as shown in Fig. 5. The principles of these method have been described in detail by Inhester (2006). The uncertainties are still quite high, because of the small difference in the aspect angles $\left(3.6^{\circ}\right)$.

\subsection{SUMER observations}

A raster scan with the $4^{\prime \prime}$ wide slit was performed in west-east direction from about $+300^{\prime \prime}$ to $-300^{\prime \prime}$. The useful slit length reached from $24 \mathrm{Mm}$ in height above the limb to approximately

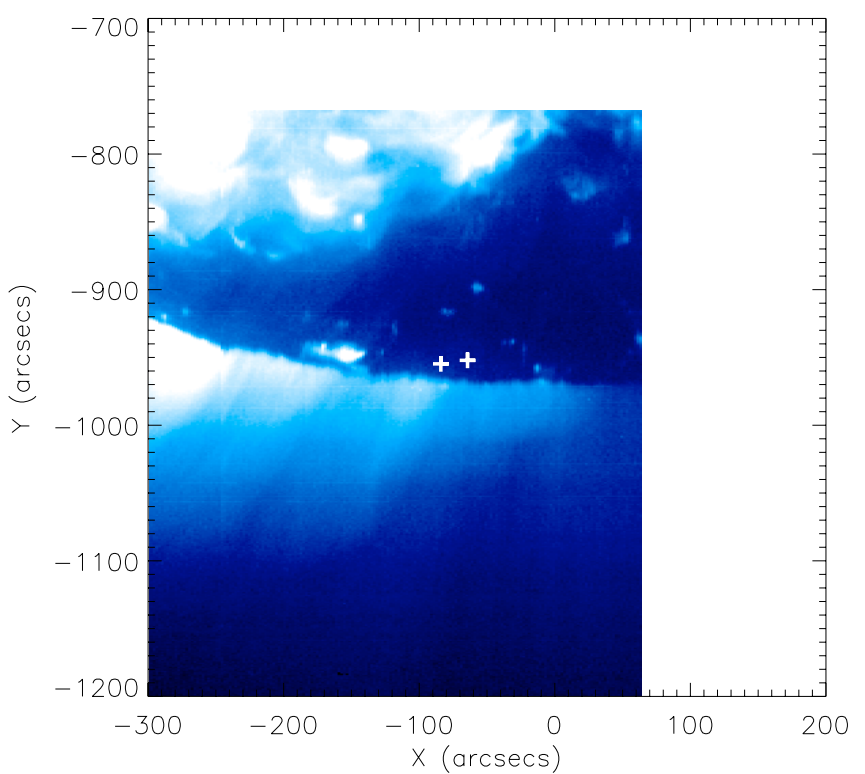

Fig. 3. The Fe XII $19.5 \mathrm{~nm}$ radiance map obtained with EIS covers a range of $373^{\prime \prime}$ in the eastern section of Fig. 1.

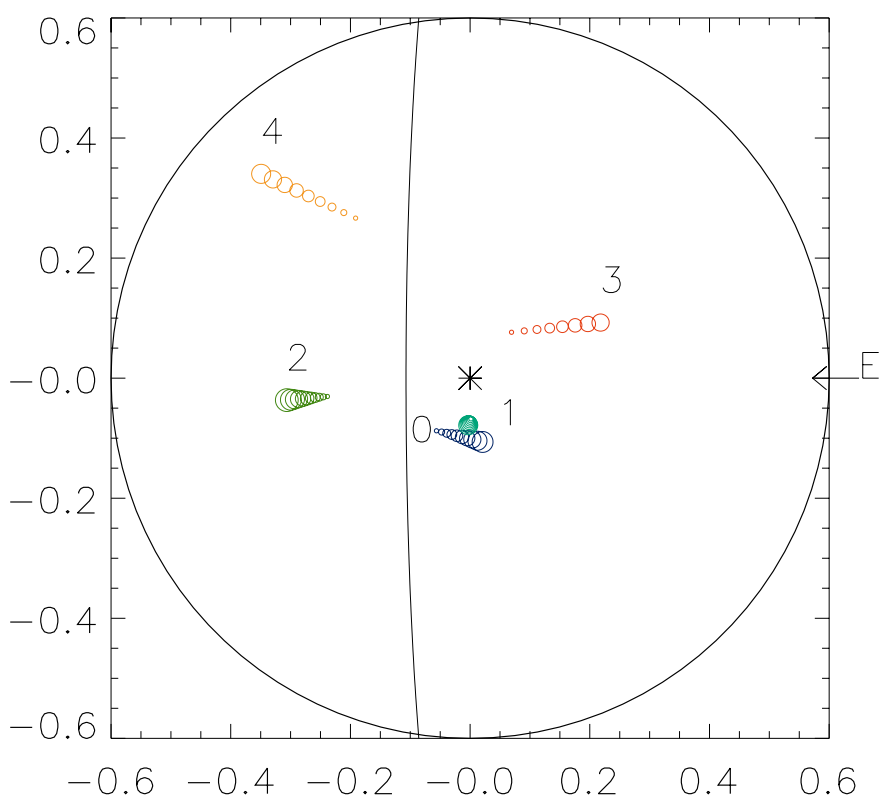

Fig. 4. Results of the 3D reconstruction in epipolar projection (view onto the southern polar region). The scales are in solar radii and the out-of-plane distances of the fitted positions are coded by the symbol size. The smallest circles denote the footpoints on the disk. The viewing direction from the Earth and the visible limb are indicated.

$200 \mathrm{Mm}$, above which noise contributions affected the line ratio calculations in particular. The prominent emission lines O VI at $103.2 \mathrm{~nm}$ and $103.8 \mathrm{~nm}$, Si VIII at $144.0 \mathrm{~nm}$ and $144.6 \mathrm{~nm}$, $\mathrm{Mg}$ IX at $70.6 \mathrm{~nm}$ and $75.0 \mathrm{~nm}, \mathrm{Ne}$ VIII at $77.0 \mathrm{~nm}, \mathrm{Mg}$ VIII at $77.2 \mathrm{~nm}$, and H I Ly $\beta$ at $102.5 \mathrm{~nm}$ were included in the spectral ranges covered. The low count rates in the interplume regions of the $\mathrm{CH}$ required a binning of the $\approx 1^{\prime \prime}$ wide pixels by a factor of eight for some of the lines to form spatial super-pixels.

The density-sensitive Si VIII line pair was used to determine the electron density, $n_{\mathrm{e}}$, averaged along the line of sight (LOS). The variation from plume to interplume regions, therefore, is underestimated. A detailed analysis of similar 


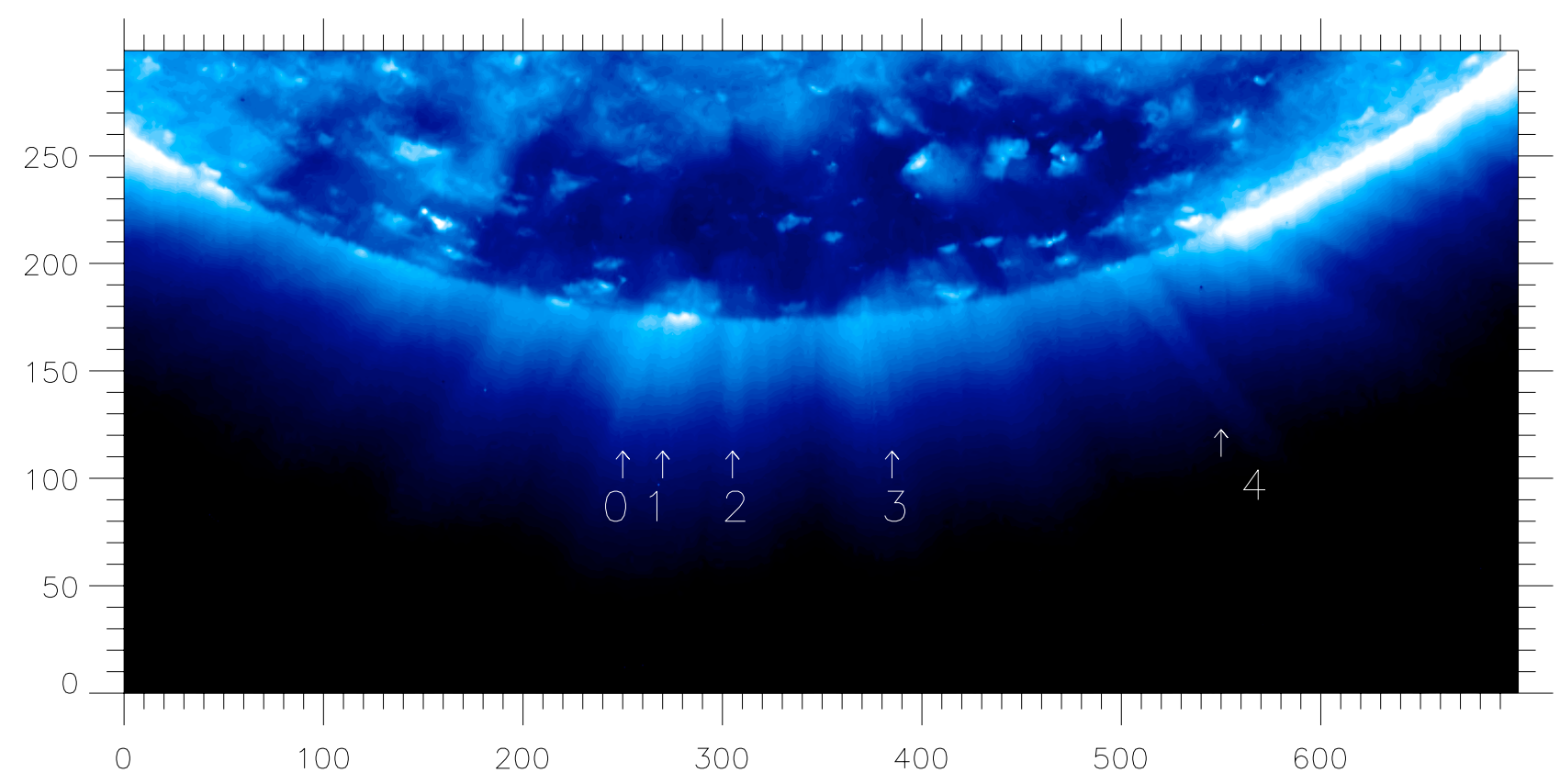

Fig. 5. Images taken by the EUVI instruments on STEREO A and B as used for a 3D reconstruction of the polar coronal plumes (differences between both images cannot be detected by visual inspection and only the A image is shown here). The locations and projected directions of five selected plumes are shown. The east-west extension is 700 pixels corresponding to $800 \mathrm{Mm}$.

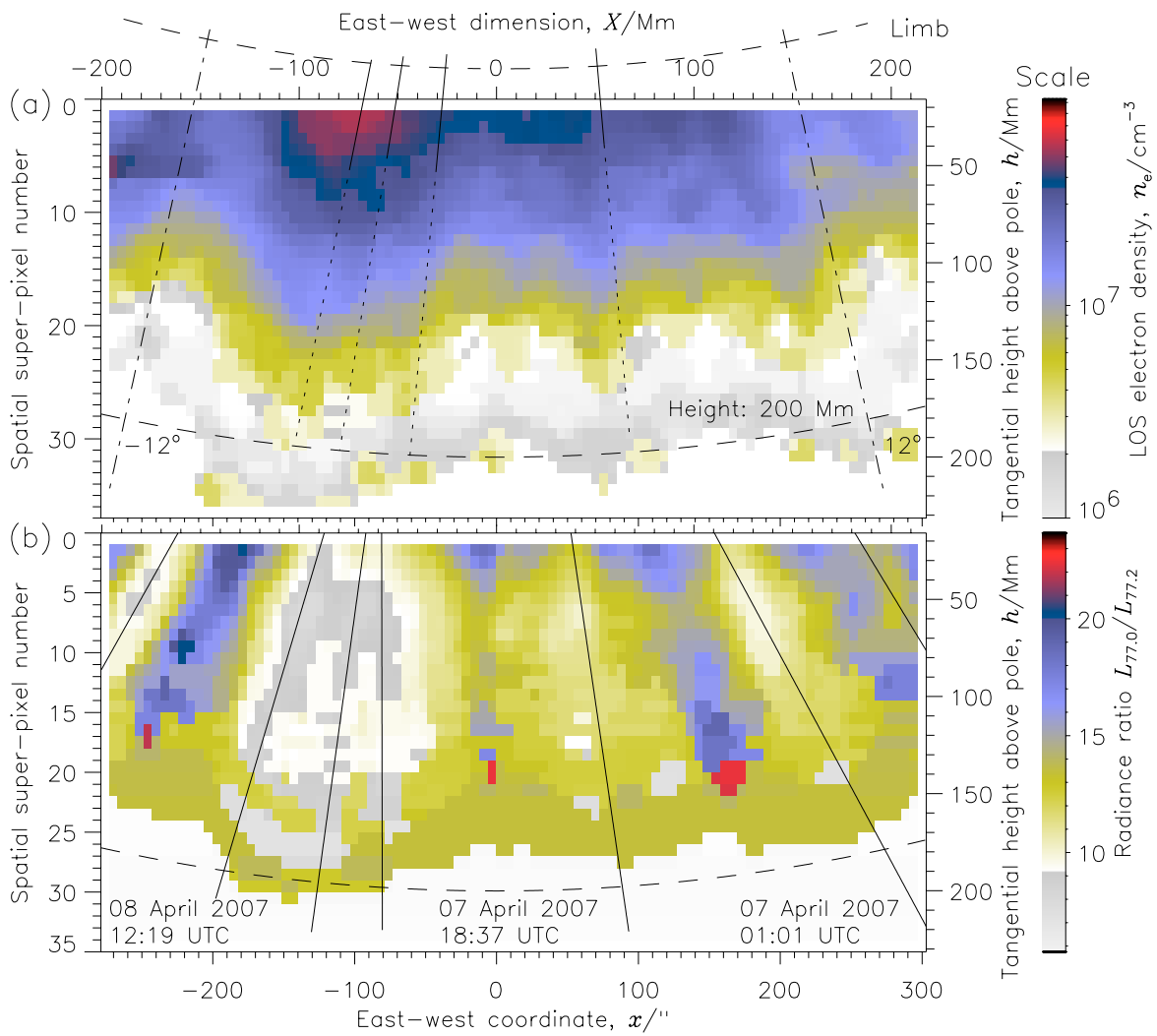

Fig. 6. SUMER observations in April 2007 in the southern $\mathrm{CH}$ of the Sun. The solar limb is indicated in the upper margin by a dashed line, and the radial directions from the centre of the disk at $\pm 12^{\circ}$ with respect to the negative $y$ axis are shown as dashed-dotted line.

a) The density-sensitive Si VIII line ratio yields an estimate of the electron density, $n_{\mathrm{e}}$. Superimposed on the density plot are, in solid lines, the projections of the 3D plume reconstructions from the EUVI observations. Plume number 4 is not within the SUMER scan range. The projections are extrapolated by dotted lines.

b) Variations in the radiance ratio $R=L_{77.0} / L_{77.2}$ are indicative of an elemental abundance anomaly caused by the difference in first ionization potential of high FIP element neon and low FIP element magnesium. The ratio $R$ exhibits very clear signatures of plume and interplume regions. The combined SUMER results on plume projections are shown as solid lines for a comparison with the EUVI results.

observations obtained in 2005 has shown that the plume density is about five times higher than that of the environment in this altitude range (Wilhelm 2006). The electron temperature, $T_{\mathrm{e}}$, in plumes is lower than in interplume regions (cf., Wilhelm et al. 1998), a tendency also found with the Mg IX temperaturesensitive line pair (not shown) in the present data. The density is shown in Fig. 6a. The insensitivity of the Si VIII ratio to scattered radiation is discussed by Wilhelm et al. (1998) and Wilhelm (2006). It is caused by the lines being barely visible on the disk (Curdt et al. 2001) and subtracted in the corona by the standard background correction. 
The contribution functions of $\mathrm{Ne}$ VIII and $\mathrm{Mg}$ VIII overlap considerably in the temperature range just below $1 \mathrm{MK}$. Variations in the radiance ratio, $R=L_{77.0} / L_{77.2}$ can, therefore, be interpreted, at least partially, as changes in the elemental abundances caused by the first ionization potential (FIP) effect (Young et al. 1999), since neon and magnesium are high and low FIP elements, respectively. The ratio $R$ was plotted in Fig. 6b, and it exhibits very clear signatures of plume and interplume regions. The same spatial area as in the upper panel was covered (obtained simultaneously). The emission of $\mathrm{Mg}$ VIII is also very faint on the disk. Consequently, the Si VIII argument with respect to the scattered radiation applies. The Ne VIII line is very prominent in the corona, but rather weak on the disk, in particular in $\mathrm{CHs}$, so that scattered radiation in the low corona is not a concern. All emission lines and the appropriate ratios have been inspected for plume structures. They allowed us to deduce composite plume projections as shown in Fig. 6b. Comparison with the radial directions from the centre of the disk at $\pm 12^{\circ}$ with respect to the negative $y$ axis indicates the super-radial expansion of the plume ensemble.

\section{Discussion and summary}

We have been able to derive the 3D plume geometry from EUVI images, although still with significant uncertainties, and to link them to other observations. Extrapolating the plume structures to the base of the corona, we could associate some plume footpoints with BPs. The temporal radiance variations in BPs are not reflected in any corresponding changes in the plumes.

The spatial radiance variations of the high FIP element neon as compared to low FIP magnesium, which outline the plume pattern, indicate abundance anomalies, although a detailed consideration of the contribution functions has still to be done.

We applied plasma diagnostics based on emission line ratios to derive the electron density, confirming that plumes have higher densities than their environment. These observations thereby favour the view that the cross-sections of plumes are restricted along the LOS direction, and thus the total area occupied by all plumes is small compared to the size of the $\mathrm{CH}$. This suggests that the plume contribution to the fast solar wind is relatively small.

There are plans for further observations with a much better stereoscopic view later in 2007 and 2008. An International Space Science Institute (ISSI) study Team lead by one of us aims to continue and extend the work presented here, motivated by the prospect of more and better observations.

Acknowledgements. The SUMER project is financially supported by DLR, CNES, NASA, and the ESA PRODEX Programme (Swiss contribution). SUMER is part of SOHO of ESA and NASA. STEREO is a NASA mission, and the Japanese mission Hinode is developed, launched, and operated by ISAS/JAXA, in collaboration with NAOJ, and NASA (USA) and STFC (UK) as international partners. The work of LF was supported by a grant from the MPS.

\section{References}

Ahmad, I. A., \& Withbroe, G. L. 1977, Sol. Phys., 53, 397

Antonucci, E., Dodero, M. A., Giordano, S., Krishnakumar, V., \& Noci, G. 2004, A\&A, 416, 749

Culhane, J. L., Harra, L. K., James, A. M., et al. 2007, Sol. Phys., 243, 19

Cirtain, J. W. L., Golub Lundquist, L., et al. 2007, Science, 318, 1580

Curdt, W., Brekke, P., Feldman, U., et al. 2001, A\&A, 375, 591

DeForest, C. E., Hoeksema, J. T., Gurman, J. B., et al. 1997, Sol. Phys., 175, 393

Gabriel, A. H., Bely-Dubau, F., \& Lemaire, P. 2003, ApJ, 589, 623

Golub, L., DeLuca, E., Austin, G., et al. 2007, Sol. Phys., 243, 63

Inhester, B. 2006, to appear as ISSI publication [arXiv: astro-ph/0612649v1]

Kosugi, T., Matsuzaki, K., Sakao, T., et al. 2007, Sol. Phys., 243, 3

Lemaire, P., Wilhelm, K., Curdt, W., et al. 1997, Sol. Phys., 170, 105

Teriaca, L., Poletto, G., Romoli, M., \& Biesecker, D. A. 2003, ApJ, 588, 566

Wang, Y.-M., Sheeley, N. R., Jr., Dere, K. P., et al. 1997, ApJ, 484, L75

Wilhelm, K. 2006, A\&A, 455, 697

Wilhelm, K., Marsch, E., Dwivedi, B. N., et al. 1998, ApJ, 500, 1023

Wülser, J.-P., Lemen, J. R., Tarbell, T. D., et al. 2004, SPIE, 5171, 111

Young, P. R., Klimchuk, J. A., \& Mason, H. E. 1999, A\&A, 350, 286 